\title{
COMBINED ALGORITHM FOR FINDING CONSERVATION LAWS AND IMPLECTIC OPERATORS FOR THE BOUSSINESQ-BURGERS NONLINEAR DYNAMICAL SYSTEM AND ITS FINITE DIMENSIONAL REDUCTIONS
}

\author{
Arkadii Kindybaliuk, Mykola Prytula \\ Ivan Franko National University of Lviv \\ Lviv, Ukraine \\ a.kindybaluk@mail.ru,mykola.prytula@gmail.com
}

\begin{abstract}
In the article the combined algorithm for finding conservation laws and implectic operators has been proposed. Using the Novikov-Bogoyavlensky method the finite dimensional reductions have been found. The structure of invariant submanifolds has been examined. Having analyzed phase portraits of Hamiltonian systems, partial periodical solutions have been found.
\end{abstract}

Keywords: nonlinear dynamical system, conservation laws, implectic operators, method of the undetermined coefficients, differential algebraic algorithm, combined algorithm, finite dimensional reduction, Hamiltonian system, exact solutions, Boussinesq-Burgers equation

\section{General scheme of the Combined Algorithm}

Conservation laws as a conception play a significant role in the numerical analysis of dynamical systems. Since conservation laws are functionals that remain constant with respect to evolution of a dynamical system, they are useful for verification of numerical schemes constructed for a such system, even if the exact solution is unknown. Moreover, according to the solitary theory the existence of infinite hierarchy of conservation laws is connected with complete integrability of a nonlinear dynamical system.

There are several ways for finding conservation laws for a nonlinear dynamical system

$$
u_{t}=K[u],
$$

where $K: M \rightarrow T(M)$ denotes the Frechét smooth tangent vector field on the smooth l-periodical manifold $M \subset C_{l}^{\infty}\left(R, R^{n}\right)$, representing a nonlinear dynamical system (1). There are two main methods: the asymptotical method (using Lax equation and asymptotical expansion) and the direct method (the main idea is solving problem for undetermined coefficients). The first method in some cases can not be 
applied to a general dynamical system (1), the second method works quickly if there are very few undetermined coefficients. When there are many unknowns, the method processing becomes slower and slower.

If functionals

$$
\gamma_{i}=\int_{x_{0}}^{x_{0}+l} \sigma_{i}[u] d x, i \in Z_{+}
$$

remain constant along vector field (1):

$$
\left.\frac{d \gamma}{d t}\right|_{K[u]} \equiv 0
$$

they are conservation laws for system (1).

Let $D(M)$ be the space of Frechét smooth functionals on manifold $M$. We define the operator grad: $D(M) \rightarrow T^{*}(M)$ by

$$
\operatorname{grad} F=\frac{\delta F}{\delta u}
$$

for $F \in D(M)$, where $\delta(\cdot) / \delta u$ is Euler variational derivative [1]

$$
\frac{\delta(\cdot)}{\delta u}=\sum_{k=0}^{\infty}(-1)^{k}\left(\frac{d}{d x}\right)^{k} \frac{\partial(\cdot)}{\partial u^{(k)}}
$$

In this paper we propose a combined algorithm for finding conservation laws (2) in a few steps:

- Step 1. Find few conservation laws by means of undetermined coefficients (direct method), sufficient for finding an implectic operator.

- Step 2. Construct first operator $\vartheta$ by means of a differential-algebraic method.

- Step 3. If operator $\vartheta$ satisfies Nöther's equation, find corresponding Hamiltonian $H_{\vartheta}$ using Hamiltonity property of the system $u_{t}=-\vartheta \operatorname{grad} H_{\vartheta}=K[u]$, we obtain the first implectic operator $\vartheta$.

- Step 4. By means of the differential-algebraic method construct second operator $\eta$.

- Step 5. Test operator $\eta$ : if operator $\eta$ satisfies Nöther's equation, then system (1) is bi-Hamiltonian with the second implectic operator $\eta$, and the recursion operator can be found as $\Lambda=\vartheta^{-1} \eta$.

This recursion operator generates an infinite hierarchy of conservation laws and their gradients are expressed as follows: $\operatorname{grad} \gamma_{i+1}=\Lambda \operatorname{grad} \gamma_{i}, i \in Z_{+}$. 
Next conservation laws are obtained explicitly, without asimptotic expansions or solving systems of linear algebraic equations. This implies fast processing for finding conservation laws.

In the next sections we will examine the application of this algorithm for the Boussinesq-Burgers nonlinear dynamical system and we will use obtained results for finding finite dimensional reductions.

\section{Problem formulation}

Let $M \subset C_{l}^{\infty}\left(R, R^{2}\right)$ be $l$-periodical smooth manifold. We consider the Boussinesq-Burgers nonlinear dynamical system [2] given on manifold $M$ :

$$
\left\{\begin{array}{l}
u_{t}=\frac{1}{2} v_{x}-2 u u_{x} \\
v_{t}=\frac{1}{2} u_{x x x}-2(u v)_{x}
\end{array}\right\}=K[u, v],
$$

where $u=u(x, t), v=v(x, t)$ are functions, $K: M \rightarrow T(M)$ denotes the Frechét smooth tangent vector field on the manifold $M$ representing the evolution of a nonlinear dynamical system (5). Setting parameter $\alpha=1$ in Broer-Kaup-Kupershmidt (BKK) system [2]

$$
\left\{\begin{array}{l}
u_{t}=-\frac{1-\alpha}{2} u_{\text {яg }}+\frac{1}{2} v_{x}-2 u u_{x} \\
v_{t}=\frac{\alpha(2-\alpha)}{2} u_{x x x}+\frac{1-\alpha}{2} v_{x x}-2(u v)_{x}
\end{array}\right\}=K[u, v],
$$

we obtain system (5). Implectic operators and recursion operator for Broer-KaupKupershmidt have been found in [2].

In this article we shall find conservation laws for (5) by means of a combined algorithm. Moreover, we construct a corresponding finite dimensional invariant submanifold for a Boussinesq-Burgers nonlinear dynamical system (5). Differential-geometric properties of this submanifold should be examined. Also we shall construct Hamiltonian system and upon its phase portrait we shall find the set of appropriate initial data to obtain periodical solutions. Furthermore we will present a phase portrait for the Hamiltonian system and show a partial exact solution.

\section{Application of the combined algorithm: conservation laws, implectic operators, recursion operator}

In this section we will use the combined algorithm proposed in the first section. Using the method of undetermined coefficients we have found the three first conservation laws. 
Theorem 1. The following functionals are conservation laws for system (5):

$$
\gamma_{0}=\int_{x_{0}}^{x_{0}+l} u d x, \gamma_{1}=\int_{x_{0}}^{x_{0}+l} v d x, \gamma_{2}=\int_{x_{0}}^{x_{0}+l} u v d x
$$

Proof. According to invariance equality (3) for functionals (1) we have to verify that $\left.\frac{d \gamma_{0}}{d t}\right|_{K[u, v]} \equiv 0,\left.\frac{d \gamma_{1}}{d t}\right|_{K[u, v]} \equiv 0,\left.\frac{d \gamma_{2}}{d t}\right|_{K[u, v]} \equiv 0$. Since manifold $M$ is $l$-periodical, we obtain $\left.\frac{d \gamma_{0}}{d t}\right|_{K[u, v]}=\left.\int_{x_{0}}^{x_{0}+l} u_{t}\right|_{K[u, v]} d x=\int_{x_{0}}^{x_{0}+l}\left(1 / 2 v_{x}-2 u u_{x}\right) d x=\left(1 / 2 v-\left.u^{2}\right|_{x_{0}} ^{x_{0}+l} \equiv 0\right.$, $\left.\frac{d \gamma_{1}}{d t}\right|_{K[u, v]}=\left.\int_{x_{0}}^{x_{0}+l} v_{t}\right|_{K[u, v]} d x=\int_{x_{0}}^{x_{0}+l}\left(1 / 2 u_{x x x}-2(u v)_{x}\right) d x=\left.\left(1 / 2 u_{x x}-u v\right)\right|_{x_{0}} ^{x_{0}+l} \equiv 0$, $\left.\frac{d \gamma_{2}}{d t}\right|_{K[u, v]}=\left.\int_{x_{0}}^{x_{0}+l}(u v)_{t}\right|_{K[u, v]} d x=\int_{x_{0}}^{x_{0}+l}\left(\left.u v_{t}\right|_{K[u, v]}+\left.v u_{t}\right|_{K[u, v]}\right) d x \equiv 0$.

Having found the three first conservation laws we can construct implectic operator by means of a differential-algebraic algorithm. Let $\partial$ denote a partial derivative with respect to variable $x: \partial=\frac{\partial}{\partial x}$.

Proposition 1. System (5) possesses implectic operator $\vartheta$ :

$$
\vartheta=\left(\begin{array}{ll}
0 & \partial \\
\partial & 0
\end{array}\right)
$$

and the corresponding Hamiltonian function is following

$$
H_{\vartheta}=\int_{x_{0}}^{x_{0}+l}\left(u^{2} v-\frac{1}{4} v^{2}+\frac{1}{4} u_{x}^{2}\right) d x
$$

Proof. This implectic operator is given in [2]. We show that we obtain this operator by means of a differential algebraic algorithm. Let us denote $H_{\eta}=H_{\eta}[u, v]=-\gamma_{2}[u, v]=\int_{x_{0}}^{x_{0}+l}(u v) d x$ and bilinear form $\langle a, b\rangle=\int_{x_{0}}^{x_{0}+l} a b d x, \forall a, b \in M$.

Using a differential algebraic algorithm we obtain the following expressions:

$H_{\eta}=-\left\langle u, v>=-\frac{1}{2}\left\langle u, v>-\frac{1}{2}<v, u>=\right.\right.$

$=\frac{1}{2}<\partial^{-1} v, u_{x}>+\frac{1}{2}<\partial^{-1} u, v_{x}>=<\sigma_{1}, u_{x}>+<\sigma_{2}, v_{x}>$, 
where $\sigma_{1}=\frac{1}{2} \partial^{-1} v, \sigma_{2}=\frac{1}{2} \partial^{-1} u$. Let us construct operators $\sigma^{\prime}, \sigma^{\prime *}, \theta_{1}^{-1}$ according to [3]: $\sigma^{\prime}=1 / 2\left(\begin{array}{cc}0 & \partial^{-1} \\ \partial^{-1} & 0\end{array}\right), \sigma^{\prime *}=-1 / 2\left(\begin{array}{cc}0 & \partial^{-1} \\ \partial^{-1} & 0\end{array}\right), \theta_{1}^{-1}=\left(\begin{array}{cc}0 & \partial^{-1} \\ \partial^{-1} & 0\end{array}\right)$. Inverse operator $\theta_{1}$ exists and it is $\vartheta=\theta_{1}=\left(\begin{array}{ll}0 & \partial \\ \partial & 0\end{array}\right)$.

Since operator $\vartheta$ satisfies Nöther's equation $\vartheta_{t}-\vartheta K^{\prime *}-K^{\prime} \vartheta=0$ operator $\vartheta$ is implectic, where operators $K^{\prime}, K^{\prime *}$ are following

$$
K^{\prime}=\left(\begin{array}{cc}
-2 \partial u & 1 / 2 \partial \\
1 / 2 \partial^{3}-2 \partial v & -2 \partial u
\end{array}\right), K^{\prime^{*}}=\left(\begin{array}{cc}
-2 u \partial & -1 / 2 \partial^{3}+2 v \partial \\
-1 / 2 \partial & 2 u \partial
\end{array}\right)
$$

With respect to Hamiltonity property of the system (5) we obtain problem

$$
\left\{\begin{array}{c}
\text { find functional } H_{\vartheta} \text { such that } \\
K[u, v]=- \text { grad }_{\vartheta} .
\end{array}\right.
$$

Thus, we have the following expressions:

$$
-\left(\begin{array}{ll}
0 & \partial \\
\partial & 0
\end{array}\right)\left(\begin{array}{l}
\delta H_{g} / \delta u \\
\delta H_{g} / \delta v
\end{array}\right)=\left(\begin{array}{l}
1 / 2 v_{x}-2 u u_{x} \\
1 / 2 u_{x x x}-2(u h) x
\end{array}\right)
$$

Expressions $\quad \delta H_{g} / \delta u=-1 / 2 u_{x x}+2 u v, \delta H_{\vartheta} / \delta v=-1 / 2 v+u^{2} \quad$ yield Hamiltonian function (8), moreover $H_{\vartheta}$ is the conservation law for system (5).

Thus we have performed yet the first three steps of the combined algorithm. We perform the next steps below.

Proposition 2. System (5) is bi-Hamiltonian, and second implectic operator is

$$
\eta=\left(\begin{array}{cc}
1 / 2 \partial & -\partial u \\
-u \partial & 1 / 2 \partial^{3}-v \partial-\partial v
\end{array}\right)
$$

and corresponding Hamiltonian is

$$
H_{\eta}=H_{\eta}[u, v]=-\gamma_{2}[u, v]=\int_{x_{0}}^{x_{0}+l}(u v) d x
$$


Proof. Let us consider Hamiltonian $H_{\vartheta}$ :

$$
\begin{gathered}
H_{\vartheta}=<u^{2} v-\frac{1}{4} v^{2}+\frac{1}{4} u_{x}^{2}, 1>=<u v, u>-\frac{1}{4}<v, v>+\frac{1}{4}<u_{x}, u_{x}>= \\
=-<\partial^{-1}(u v), u_{x}>+\frac{1}{4}<u_{x}, u_{x}>+\frac{1}{4}<\partial^{-1} v, v_{x}>= \\
=<\frac{1}{4} \partial^{-1} v-\partial^{-1}(u v), u_{x}>+<\frac{1}{4} \partial^{-1} v, v_{x}>.
\end{gathered}
$$

According to the proof of Theorem 2 and [3] we obtain operators $\sigma^{\prime}, \sigma^{\prime^{*}}, \theta_{2}^{-1}$ :

$$
\begin{gathered}
\sigma^{\prime}=\left(\begin{array}{cc}
\frac{1}{4} \partial-\partial^{-1} v & -\partial^{-1} u \\
0 & \frac{1}{4} \partial^{-1}
\end{array}\right), \sigma^{\prime^{*}}=\left(\begin{array}{cc}
-\frac{1}{4} \partial+v \partial^{-1} & 0 \\
u \partial^{-1} & -\frac{1}{4} \partial^{-1}
\end{array}\right), \\
\theta_{2}^{-1}=\sigma^{\prime}-\sigma^{*^{*}}=\left(\begin{array}{cc}
\frac{1}{2} \partial-\partial^{-1} v-v \partial^{-1} & -\partial^{-1} u \\
-u \partial^{-1} & \frac{1}{2} \partial^{-1}
\end{array}\right) .
\end{gathered}
$$

Operator $\eta$ has been obtained from the relationship $\eta=\vartheta \theta_{2}^{-1} \vartheta$ :

$$
\begin{gathered}
\eta=\vartheta \theta_{2}^{-1} \vartheta=\left(\begin{array}{ll}
0 & \partial \\
\partial & 0
\end{array}\right)\left(\begin{array}{cc}
\frac{1}{2} \partial-\partial^{-1} v-v \partial^{-1} & -\partial^{-1} u \\
-u \partial^{-1} & \frac{1}{2} \partial^{-1}
\end{array}\right)\left(\begin{array}{ll}
0 & \partial \\
\partial & 0
\end{array}\right)= \\
=\left(\begin{array}{cc}
\frac{1}{2} \partial & -\partial u \\
-u \partial & \frac{1}{2} \partial^{3}-v \partial-\partial v
\end{array}\right)
\end{gathered}
$$

Since operator $\eta$ satisfies Nöther's equation $\eta_{t}-\eta K^{\prime *}-K^{\prime} \eta=0$ operator $\eta$ is implectic, and system (5) is bi-Hamiltonian.

Theorem 2. System (5) possesses an infinite hierarchy of conservation laws $\left\{\gamma_{i}\right\}, i \in \mathbf{Z}_{+}$, which are generated by a recursion operator

$$
\Lambda=\left(\begin{array}{cc}
-\partial^{-1} u \partial & \frac{1}{2} \partial^{2}-\partial^{-1} v \partial-v \\
\frac{1}{2} & -u
\end{array}\right),
$$

with a gradient relationship $\operatorname{grad} \gamma_{i+1}=\Lambda \operatorname{grad} \gamma_{i}, i \in \mathbf{Z}_{+}$. 
Proof. Since operators $\vartheta$ and $\eta$ are implectic and expression

$$
\vartheta \operatorname{grad} \gamma_{i+1}=\eta \operatorname{grad} \gamma_{i}
$$

holds for all $i \in \mathbf{Z}_{+}[4]$. Having multiplied (12) by $\vartheta^{-1}$ we obtain

$$
\operatorname{grad} \gamma_{i+1}=\vartheta^{-1} \operatorname{\eta grad}_{i}, i \in \mathbf{Z}_{+} .
$$

hence $\Lambda=\vartheta^{-1} \eta$ is a recursion operator. However

$$
\vartheta^{-1}=\left(\begin{array}{cc}
0 & \partial^{-1} \\
\partial^{-1} & 0
\end{array}\right)
$$

we obtain recursion operator:

$$
\Lambda=\vartheta^{-1} \eta=\left(\begin{array}{cc}
0 & \partial^{-1} \\
\partial^{-1} & 0
\end{array}\right)\left(\begin{array}{cc}
\frac{1}{2} \partial & -\partial u \\
-u \partial & \frac{1}{2} \partial^{3}-v \partial-\partial v
\end{array}\right)=\left(\begin{array}{cc}
-\partial^{-1} u \partial & \frac{1}{2} \partial^{2}-\partial^{-1} v \partial-v \\
\frac{1}{2} & -u
\end{array}\right)
$$

Using these obtained results, we have found the next conservation laws in the hierarchy

$$
\begin{gathered}
\gamma_{4}=\int_{x_{0}}^{x_{0}+l}\left(-u^{3} v+\frac{3}{4} u v^{2}-\frac{3}{4} u u_{x}^{2}+\frac{1}{4} u_{x} v_{x}\right) d x \\
\gamma_{5}=\int_{x_{0}}^{x_{0}+l}\left(u^{4} v-\frac{3}{2} u^{2} v^{2}+\frac{1}{8} v^{3}+\frac{3}{2} u^{2} u_{x}-\frac{5}{8} v u_{x}^{2}-u u_{x} v_{x}+\frac{1}{16}\left(v_{x}^{2}-u_{x x}^{2}\right)\right) d x .
\end{gathered}
$$

Functional $\gamma_{4}$ we will use for four-dimensional reduction of the system (5).

\section{Finite dimensional reduction on two-dimensional invariant submanifold}

Finding the appropriate set of initial conditions for an infinite dimensional conservative nonlinear dynamical system for special solutions like a solitary wave on periodical functional manifolds is a burning issue for numerical investigation of such systems [5] and connected with some difficulties.

We will use the finite dimensional reduction method by Novikov-Bogoyavlensky [6] for constructing exact solutions for a nonlinear dynamical system by means of generalizations presented in [1,7-10]. 
Let us consider the invariant submanifold $M^{2} \in M$ as the set of critical points of the following Lagrangian functional $\mathrm{L}_{2} \in D(M)$ :

$$
\mathrm{L}_{2}[u, v]:=\int_{x_{0}}^{x_{0}+l}\left[c_{\eta} u v+c_{\vartheta}\left(u^{2} v-\frac{1}{4} v^{2}+\frac{1}{4} u_{x}^{2}\right)\right] d x,
$$

where $c_{\eta}, c_{\vartheta}$ are arbitrary constants.

According to Lax proposition $[4,10]$ a finite dimensional functional submanifold for dynamical system (5) is determined by $M^{2}:=\left\{(u, v) \in M: \operatorname{grad} \mathrm{L}_{2}[u, v]=0\right\}$.

Having evaluated $\operatorname{grad} \mathbf{L}_{2}$ using (4) we obtain

$$
\begin{array}{r}
M^{2}:=\left\{(u, v) \in M: \frac{\delta \mathrm{L}_{2}}{\delta u}=c_{\eta} v+2 c_{\vartheta} u v-\frac{1}{2} c_{\vartheta} u_{x x}=0,\right. \\
\left.\frac{\delta \mathrm{L}_{2}}{\delta v}=c_{\eta} u+c_{\vartheta} u^{2}-\frac{1}{2} c_{\vartheta} v=0\right\} .
\end{array}
$$

Constraints (14) yield $v=\frac{2 c_{\eta}}{c_{\vartheta}} u+2 u^{2}$. Using the Gelfand-Dickey relationship [3], the differential of (13) is:

$$
d \mathrm{~L}_{3}=\left(c_{\eta} v+2 c_{\vartheta} u v-\frac{1}{2} c_{\vartheta} u_{x x}\right) d u+\left(c_{\eta} u+c_{\vartheta} u^{2}-\frac{1}{2} c_{\vartheta} v\right) d v+\frac{d}{d x}\left(\frac{1}{2} c_{\vartheta} u_{x} d u\right)
$$

which yields 1 -form $[3,7] \alpha^{(1)}:=\frac{1}{2} c_{9} u_{x} d u$.

We determine symplectic structure $\omega^{(2)}$ as an outer differentiation of 1-form $\alpha^{(1)}$ :

$$
\omega^{(2)}:=d \alpha^{(1)}=d\left(\frac{1}{2} c_{9} u_{x}\right) \wedge d u
$$

on the submanifold $M^{2} \in M$.

However submanifold $M^{2} \in M$ is simplectic with the structure (15), we introduce canonical variables on $M^{2} \in M$ :

$$
p:=\frac{1}{2} c_{\vartheta} u_{x}, q:=u
$$

Elements of the phase space $u, v$ in canonical variables (15) of submanifold $M^{2} \subset M$ are

$$
v=\frac{2 c_{\eta}}{c_{\vartheta}} q+2 q^{2}
$$


Hamiltonian $h_{2}^{(x)}$ which corresponds to vector field $d / d x$ has been found from the determining relationship $\frac{d h_{2}^{(x)}}{d x}=-\left(\frac{\delta \mathrm{L}_{2}}{\delta u} u_{x}+\frac{\delta \mathrm{L}_{2}}{\delta v} v_{x}\right)$.

Hence, the founded Hamiltonian $h_{2}^{(x)}$ in local coordinates is:

$$
h_{2}^{(x)}=-c_{\eta} u v-c_{\vartheta} u^{2} v+\frac{1}{4} c_{\vartheta} v^{2}+\frac{1}{4} u_{x}^{2},
$$

and in canonical coordinates (16) of submanifold $M^{2} \subset M$ has following form

$$
h_{2}^{(x)}(q, p)=\frac{1}{c_{\vartheta}} p^{2}-\frac{c_{\eta}^{2}}{c_{\vartheta}} q^{2}-2 c_{\eta} q^{3}-c_{\vartheta} q^{4} .
$$

According to [7] the Hamiltonian system has been obtained

$$
\left\{\begin{array}{l}
\frac{d q}{d x}=\frac{2 p}{c_{\vartheta}}, \\
\frac{d p}{d x}=\frac{2 c_{\eta}^{2} q}{c_{\vartheta}}+6 c_{\eta} q^{2}+4 c_{\vartheta} q^{3},
\end{array}\right.
$$

which has three fixed points in a phase space: two hyperbolic points with coordinates $\left(q_{0}, p_{0}\right)=(0,0),\left(q_{0}, p_{0}\right)=\left(-\frac{c_{\eta}}{c_{\vartheta}}, 0\right)$ and elliptic point with coordinates $\left(q_{0}, p_{0}\right)=\left(-\frac{c_{\eta}}{2 c_{\vartheta}}, 0\right)$

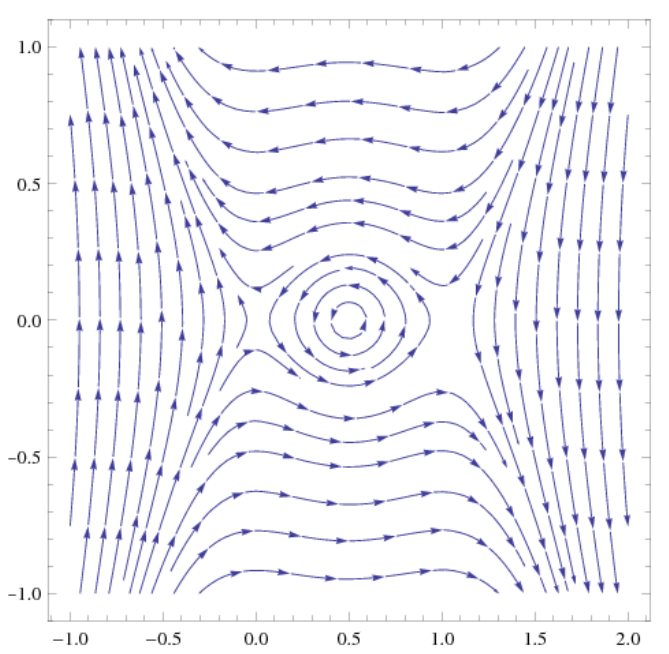

Fig. 1. Phase portrait of the system (19) with $c_{\eta}=1, c_{\vartheta}=-1$ 
The phase portrait of the system (19) provides important information for identifying the set of initial conditions for periodical solutions and is shown in Figure 1. If initial data for the system (19) are taken from the internal region of limit cycle of the system (19) we will obtain a periodical solution for problem (5).

Vector field $d / d t$ is Hamiltonian too. The Hamiltonian $h_{2}^{(t)}$, which corresponds to vector field $d / d t$ has been found from the determining relationship

$$
\frac{d h_{2}^{(t)}}{d x}=-\left.\left(\frac{\delta \mathrm{L}_{2}}{\delta u} u_{t}+\frac{\delta \mathrm{L}_{2}}{\delta v} v_{t}\right)\right|_{K[u, v]} .
$$

and $h_{2}^{(t)}$ has the following form in local coordinates:

$$
h_{2}^{(t)}=2 c_{\eta} u^{2} v+2 c_{\vartheta} u^{3} v-\frac{c_{\eta}}{4} v^{2}-c_{\vartheta} u v^{2}+\frac{c_{\eta}}{4} u_{x}^{2}-\frac{c_{\eta}}{2} u u_{x x}-\frac{c_{\vartheta}}{2} u^{2} u_{x x}+\frac{c_{\vartheta}}{4} u_{x x} v,
$$

which in canonical variables (16) of invariant submanifold $M^{2} \in M$ is

$$
h_{2}^{(t)}(q, p)=\left(\frac{c_{\eta}}{c_{\vartheta}}\right) h_{2}^{(x)}(q, p)=\frac{c_{\eta}}{c_{\vartheta}^{2}} p^{2}-\frac{c_{\eta}^{3}}{c_{\vartheta^{2}}} q^{2}-2 \frac{c_{\eta}^{2}}{c_{\vartheta}} q^{3}-c_{\eta} q^{4} .
$$

Finally, we obtain Hamiltonian system:

$$
\left\{\begin{array}{l}
\frac{d q}{d t}=\frac{2 c_{\eta} p}{c_{9}^{2}} \\
\frac{d p}{d t}=\frac{2 c_{\eta}^{3} q}{c_{\vartheta}^{2}}+\frac{6 c_{\eta}^{2} q^{2}}{c_{\vartheta}}+4 c_{\eta} q^{3},
\end{array}\right.
$$

which possesses three fixed points in the phase space: the same as for system (19).

The phase portrait of system (22) is the same as the portrait of system (19). If we choose initial data for system (22) from the region which provides periodical solution we will obtain periodical solution.

Theorem 3. Boussinesq-Burgers nonlinear dynamical system (5) reduced on the invariant two dimensional submanifold $M^{2} \subset M$ is exactly equivalent to the set of two commuting canonical Hamiltonian flows (19) and (22) that are completely integrable by quadratures systems. The corresponding Hamiltonian functions are given by expressions (18) and (21).

\section{Partial solutions on the two-dimensional invariant submanifold}

We obtain a partial exact solution of the dynamical system (5) via layering vector fields $d / d x$ (19) and $d / d t$ (22). 
Exact solutions for systems (19) and (22) can be written in the following form:

$$
x-x_{0}=\int_{q_{0}}^{q} \frac{\left|c_{\vartheta}\right| d q}{2 \sqrt{c_{\eta}^{2} q^{2}+2 c_{\vartheta} q^{3}+2 c_{\vartheta}^{2} q^{4}+\beta}}, t-t_{0}=\int_{q_{0}}^{q} \frac{1}{2} \sqrt{\frac{c_{\vartheta}^{3}}{c_{\eta}^{3} q^{2}+2 c_{\eta} c_{\vartheta} q^{3}+2 c_{\eta} c_{\vartheta}^{2} q^{4}+\beta}} d q,
$$

where $x_{0}, t_{0}, q_{0}$ denote arbitrary real values and $\beta$ is arbitrary real parameter.

Let coefficients of the system (5) and coefficients in the Lagrange functional (13) are following $c_{\eta}=1, c_{\vartheta}=-1$. We integrate system (19), which corresponds vector field $d / d x$ on the domain $x \in(0,10]$ with the step $\Delta x=0.1$ by variable $x$ with initial data $\left(q_{0}=0.5, p_{0}=0.2\right)$, which are in the limit cycle of system (19). However, the following relationships

$$
u=q, v=2 q(q-1)
$$

hold on invariant submanifold $M^{2} \subset M$, then in the initial moment of time $t_{0}=0$ solutions $u(x, 0), v(x, 0)$ of nonlinear dynamical system (5) are shown in Figure 2.
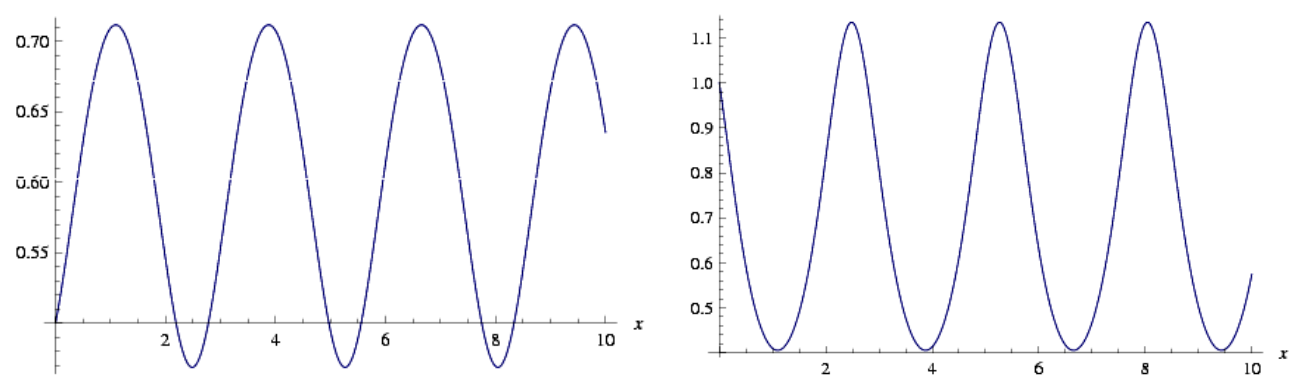

Fig. 2. Solution $u(x, 0)$ and $v(x, 0)$ of the system (5) with $c_{\eta}=1, c_{\vartheta}=-1$ in the initial moment of time $t_{0}=0$

We integrate the system (22) associated with vector field $d / d t$ on the domain $t \in(0,10]$ with step $\Delta t=0.1$ using the following initial data $\left(q_{0}=0.5, p_{0}=0.2\right)$ which are in the limit cycle of system (22). Hence we obtain time evolution of the solution in the point $x=0: q(0, t), p(0, t)$.

At every moment of time $t=t_{k}$ we integrate system (19) with initial data

$$
\left(q_{0}=q\left(0, t_{k}\right), p_{0}=p\left(0, t_{k}\right)\right)
$$

to obtain solutions $u(x, t), v(x, t)$.

Using relationships (23) we obtain exact solutions of nonlinear dynamical system (5) for $u=u(x, t)$ and $v=v(x, t)$ which are given in Figure 3. 

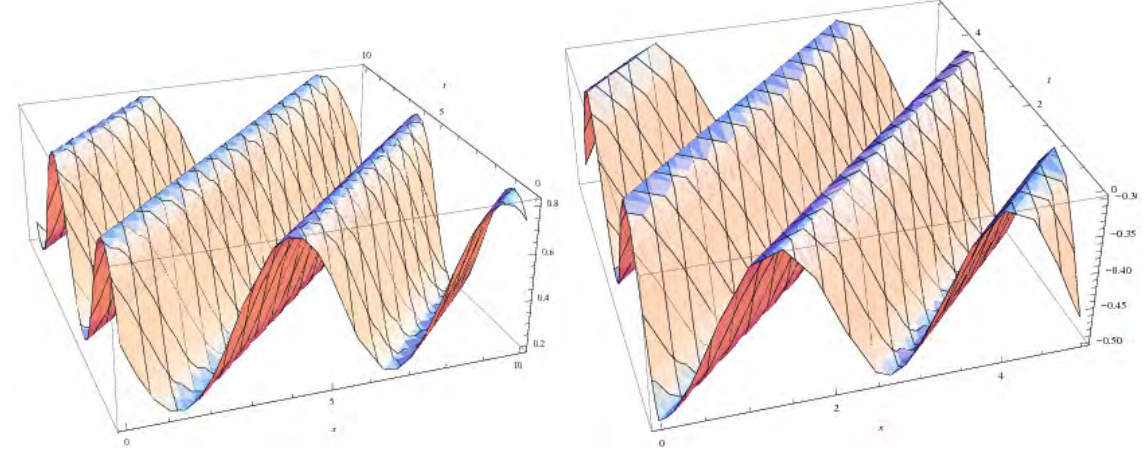

Fig. 3. Solutions $u=u(x, t)$ and $v=v(x, t)$ of the system (5) with $c_{\eta}=1, c_{\vartheta}=-1$

\section{Finite dimensional reduction on the four-dimensional invariant submanifold}

Let us consider the following Lagrange functional

$$
\mathrm{L}_{4}[u, v]:=\int_{x_{0}}^{x_{0}+l}\left[c_{\eta} u v+c_{\vartheta}\left(u^{2} v-\frac{1}{4} v^{2}+\frac{1}{4} u_{x}^{2}\right)+c_{4}\left(-u^{3} v+\frac{3}{4} u v^{2}-\frac{3}{4} u u_{x}^{2}+\frac{1}{4} u_{x} v_{x}\right)\right] d x .
$$

Similar to the first case, we find invariant submanifold $M^{4} \subset M$ : $M^{4}=\left\{(u, v) \in M: \operatorname{grad} \mathbf{L}_{4}[u, v]=0\right\}$ as fixed points of Lagrange functional (24):

$$
M^{4}=\left\{\begin{array}{l}
(u, v) \in M: c_{\eta} v+2 c_{9} u v-3 c_{4} u^{2} v+ \\
+\frac{3}{4} c_{4} v^{2}+\frac{3}{4} c_{4} u_{x}^{2}-\frac{1}{2} u_{x}^{2}+\frac{3}{2} c_{4} u u_{x x}-\frac{1}{4} c_{4} v_{x x}=0 \\
c_{\eta} u+c_{\vartheta} u^{2}-c_{4} u^{3}-\frac{1}{2} c_{9} v+\frac{3}{4} u v-\frac{1}{4} c_{4} u_{x x}=0
\end{array}\right\} .
$$

Thus, on manifold (25) exists symplectic structure $\omega^{(2)}=d p_{1} \wedge d q_{1}+d p_{2} \wedge d q_{2}$ in the following form

$$
\omega^{(2)}=d\left(\frac{1}{2} c_{\vartheta} u_{x}+c_{4}\left(-\frac{3}{2} u u_{x}+\frac{1}{4} v_{x}\right)\right) \wedge d u+d\left(\frac{1}{4} c_{4} u_{x}\right) \wedge d v
$$

Canonic Hamiltonian variables $\left\{p_{1}, q_{1}, p_{2}, q_{2}\right\}$ are

$$
p_{1}=\frac{1}{2} c_{9} u_{x}+c_{4}\left(-\frac{3}{2} u u_{x}+\frac{1}{4} v_{x}\right), q_{1}=u, p_{2}=\frac{1}{4} c_{4} u_{x}, q_{2}=v
$$

on submanifold $M^{4} \subset M$. 
Elements $\left\{u, v, u_{x}, v_{x}, u_{x x}, v_{x x}\right\}$ can be rewritten on submanifold $M^{4} \subset M$ as follows:

$$
\begin{gathered}
u=q_{1}, v=q_{2}, u_{x}=\frac{4}{c_{4}} p_{2}, v_{x}=\frac{4}{c_{4}^{2}}\left(c_{4} p_{1}-2 c_{\vartheta} p_{2}+6 c_{4} p_{2} q_{1}\right), \\
u_{x x}=\frac{2}{c_{4}^{2}}\left(2 c_{\eta} q_{1}+2 c_{\vartheta} q_{1}^{2}-2 c_{4} q_{1}^{3}-c_{\vartheta} q_{2}+3 c_{4} q_{1} q_{2}\right), \\
u_{x x}=\frac{1}{c_{4}^{2}}\left(48 p_{2}^{2}-8 c_{\eta} c_{\vartheta} q_{1}+24 c_{4} c_{\eta} q_{1}^{2}-8 c_{\vartheta}^{2} q_{1}^{2}+32 c_{4} c_{\vartheta} q_{1}^{3}-24 c_{4}^{2} q_{1}^{4}+4 c_{4} c_{\eta} q_{2}+\right. \\
\left.+4 c_{9}^{2} q_{2}-16 c_{4} c_{\vartheta} q_{1} q_{2}+24 c_{4}^{2} q_{1}^{2} q_{2}+3 c_{4}^{2} q_{2}^{2}\right)
\end{gathered}
$$

Hamiltonian functions $h_{4}^{(x)}$ and $h_{4}^{(t)}$ can be obtained by determining relationships: $\frac{d h_{4}^{(x)}}{d x}=-\left(\frac{\delta \mathrm{L}_{4}}{\delta u} u_{x}+\frac{\delta \mathrm{L}_{4}}{\delta v} v_{x}\right), \frac{d h_{4}^{(t)}}{d x}=-\left.\left(\frac{\delta \mathrm{L}_{4}}{\delta u} u_{t}+\frac{\delta \mathrm{L}_{4}}{\delta v} v_{t}\right)\right|_{K[u, v]}$

which have the following form in the canonical coordinates of submanifold $M^{4} \subset M$ :

$$
\begin{aligned}
h_{4}^{(x)}= & \frac{4 p_{1} p_{2}}{c_{4}}-\frac{4 c_{\vartheta} p_{2}^{2}}{c_{4}^{2}}+\frac{12 p_{2}^{2} q_{1}}{c_{4}}-c_{\eta} q_{1} q_{2}-c_{\vartheta} q_{1}^{2} q_{2}+c_{4} q_{1}^{3} q_{2}+\frac{c_{\vartheta} q_{2}^{2}}{4}-\frac{3}{4} c_{4} q_{1} q_{2}^{2} \\
& h_{4}^{(t)}=\frac{p_{1}^{2}}{c_{4}}-\frac{4 c_{\vartheta} p_{1} p_{2}}{c_{4}^{2}}+\frac{4 c_{\eta} p_{2}^{2}}{c_{4}^{2}}+\frac{4 c_{\vartheta}^{2} p_{2}^{2}}{c_{4}^{3}}+\frac{4 p_{1} p_{2} q_{1}}{c_{4}}-\frac{8 c_{\vartheta} p_{2}^{2} q 1}{c_{4}^{2}}-\frac{c_{\eta}^{2} q_{1}^{2}}{c_{4}}- \\
& \frac{2 c_{\eta} c_{\vartheta} q_{1}^{3}}{c_{4}}+2 c_{\eta} q_{1}^{4}-\frac{c_{9}^{2} q_{1}^{4}}{c_{4}}+2 c_{\vartheta} q_{1}^{5}-c_{4} q_{1}^{6}+\frac{2 p_{2}^{2} q_{2}}{c_{4}}+\frac{c_{\eta} c_{\vartheta} q_{1} q_{2}}{c 4}- \\
& c_{\vartheta} q_{1}^{2} q_{2}+\frac{c_{9}^{2} q_{1}^{2} q_{2}}{c_{4}}-2 c_{\vartheta} q_{1}^{3} q_{2}+c_{4} q_{1}^{4} q_{2}-\frac{c_{\eta} q_{2}^{2}}{4}-\frac{c_{\vartheta}^{2} q_{2}^{2}}{4 c_{4}}+1 / 2 c_{\vartheta} q_{1} q_{2}^{2}-\frac{c_{4} q_{2}^{3}}{8}
\end{aligned}
$$

As a result, we have reduced our Boussinesq-Burgers nonlinear dynamical system (5) on the constructed four dimensional invariant submanifold $M^{4} \subset M$.

The system for the $d / d x$ is obtained from (27) and provides information for initial data for the Cauchy problem

$$
\left\{\begin{array}{l}
\frac{d q_{1}}{d x}=\frac{4 p_{2}}{c_{4}} \\
\frac{d p_{1}}{d x}=-\frac{12 p_{2}^{2}}{c 4}+c_{\eta} q_{2}+2 c_{\vartheta} q_{1} q_{2}-3 c_{4} q_{1}^{2} q_{2}+\frac{3 c_{4} q_{2}^{2}}{4} \\
\frac{d q_{2}}{d x}=\frac{4 p_{1}}{c_{4}}-\frac{8 c_{\vartheta} p_{2}}{c_{4}^{2}}+\frac{24 p_{2} q_{1}}{c_{4}} \\
\frac{d p_{2}}{d x}=c_{\eta} q_{1}+c_{\vartheta} q_{1}^{2}-c_{4} q_{1}^{3}-\frac{c_{\vartheta} q_{2}}{2}+\frac{3 c_{4} q_{1} q_{2}}{2}
\end{array}\right.
$$


The system for the $d / d t$ is following:

$$
\left\{\begin{array}{l}
\frac{d q_{1}}{d t}=\frac{2 p_{1}}{c_{4}}-\frac{4 c_{\vartheta} p_{2}}{c_{4}^{2}}+\frac{4 p_{2} q_{1}}{c_{4}} \\
\frac{d p_{1}}{d t}=-\frac{4 p_{1} p_{2}}{c_{4}}+\frac{8 c_{\vartheta} p_{2}^{2}}{c_{4}^{2}}+\frac{2 c_{\eta}^{2} q_{1}}{c_{4}}+\frac{6 c_{\eta} c_{\vartheta} q_{1}^{2}}{c_{4}}-8 c_{\eta} q_{1}^{3}+ \\
+\frac{4 c_{\vartheta}^{2} q_{1}^{3}}{c_{4}}-10 c_{\vartheta} q_{1}^{4}+6 c_{4} q_{1}^{5}-\frac{c_{\eta} c_{\vartheta} q_{2}}{c_{4}}+2 c_{\eta} q_{1} q_{2}-\frac{2 c_{\vartheta}^{2} q_{1} q_{2}}{c_{4}}+ \\
+6 c_{\vartheta} q_{1}^{2} q_{2}-4 c_{4} q_{1}^{3} q_{2}-\frac{c_{\vartheta} q_{2}^{2}}{2}, \\
\frac{d q_{2}}{d t}=-\frac{4 c_{\vartheta} p_{1}}{c_{4}^{2}}+\frac{8 c_{\eta} p_{2}}{c_{4}^{2}}+\frac{8 c_{\vartheta}^{2} p_{2}}{c_{4}^{3}}+\frac{4 p_{1} q_{1}}{c_{4}}-\frac{16 c_{\vartheta} p_{2} q_{1}}{c_{4}^{2}}+\frac{4 p_{2} q_{2}}{c_{4}} \\
\frac{d p_{2}}{d t}=-\frac{2 p_{2}^{2}}{c_{4}}-\frac{c_{\eta} c_{\vartheta} q_{1}}{c_{4}}+c_{\eta} q_{1}^{2}-\frac{c_{\vartheta}^{2} q_{1}^{2}}{c_{4}}+2 c_{\vartheta} q_{1}^{3}-c_{4} q_{1}^{4}+ \\
\frac{c_{\eta} q_{2}}{2}+\frac{c_{\vartheta}^{2} q_{2}}{2 c_{4}}-c_{\vartheta} q_{1} q_{2}+\frac{3 c_{4} q_{2}^{2}}{8} .
\end{array}\right.
$$

Theorem 4. Boussinesq-Burgers nonlinear dynamical system (5) reduced on the invariant four-dimensional submanifold $M^{4} \subset M$ is exactly equivalent to the set of two commuting canonical Hamiltonian flows (29) and (30) that are completely integrable by quadratures systems. The corresponding Hamiltonian functions are given by expressions (27) and (28).

\section{Conclusions}

Thus, in this paper we present a combined algorithm for finding conservation laws and an implectic operator. This method has several advantages: having found few conservation laws, we can obtain implectic operators and furthermore construct a recursion operator for explicit evaluation of the conservation laws. Moreover, we have shown existence of the infinite hierarchy of conservations laws for Boussinesq-Burgers nonlinear dynamical system. Using conservation laws, the finite dimensional reduction on invariant two-dimensional and four-dimensional submanifolds has been performed. Initial conditions for periodical solutions for dynamical system have been found. 


\section{References}

[1] Prykarpatsky A.K., Finite-dimensional reductions of conservative dynamical systems and numerical analysis, A.K. Prykarpatsky, S. Brzychczy, V.Hr. Samoylenko, Ukr. Math. Journ. 2001, 53. 2, 220-228.

[2] Tao Chen, The generalized Broer-Kaup-Kupershmidt system and its Hamiltonian extension, Tao Chen, Li-Li Zhu, Lei Zhang, Applied Mathematical Sciences 2011, 5(76), 3767-3780.

[3] Prykarpatsky A.K., Algebraic Integrability of Nonlinear Dynamical Systems on Manifolds: Classical and Quantum Aspects, A.K. Prykarpatsky, I.V. Mykytiuk, Kluwer, Netherlands 1999, $560 \mathrm{p}$.

[4] Hentosh O.E., Differential-geometric and Lie-algebraic Foundations of Integrable Nonlinear Dynamical Systems on Functional Manifolds, O.E. Hentosh, M.M. Prytuka, A.K. Prykarpatsky, Publish Center LNU Franko, Lviv 2006, 408 p. (in Ukrainian).

[5] Arnold V.I., Mathematical Methods of Classic Mechanics, Nauka, M.: 1979, 431 p. (in Russian).

[6] Bogoyavlensky O.I., On the connection of hamiltonian formalisms for stationary and nonstationary problems, O.I. Bogoyavlensky, S.P. Novikov, Functional Analysis and Its Applications 1976, 10, 1, 9-15 (in Russian).

[7] Blackmore D., Nonlinear Dynamical Systems of the Mathematical Physics: Spectral and Differential-geometrical Integrability Analysis, D. Blackmore, A.K. Prykarpatsky, V. Hr. Samoylenko, World Scientific Publ., NJ, USA 2011, 564 p.

[8] Mytropolsky Ju.A., Integrable dynamical systems: spectral and differential-algebraic aspects, Ju.A. Mytropolsky, N.N. Bogoliubov, A.K. Prykarpatsky, V.Gr. Samoylenko, Nauk. Dumka, K.: 1987, 296 p. (in Russian).

[9] Prykarpatsky A.K., On the one construction of finite dimensional reductions on functional manifolds, A.K. Prykarpatsky, O.G. Bihun, Math. Methods and Phis.-mech. Fields, 48, 1, 7-14 (in Ukrainian).

[10] Prykarpatsky A.K., Algebraic Aspects of Integrability of Dynamical Systems on Manifolds, A.K. Prykarpatsky, I.V. Mykytiuk, A.M Samoylenko, Nauk. Dumka, K., 1991, 288 p. (in Russian).

[11] Samoylenko A.M., Algebraic-analytical Aspects of Complete Integrable Systems and Their Perturbations, A.M. Samoylenko, Ya.A. Prykarpatsky, Institute of Mathematics of NAN Ukraine, K.: 2002, 238 p. (in Ukrainian). 
